\title{
DYNAMIC IMAGING OF TUMOR PERFUSION USING CONTRAST ENHANCED ULTRASOUND: IN VIVO RESULTS
}

\author{
Juan Du, John R. Ballard, Jeunghwan Choi, John C. Bischof and Emad S. Ebbini* \\ University of Minnesota \\ College of Science and Engineering \\ Minneapolis, MN 55455 \\ \{duxxx134, ball0250, choi0158, bischof, emad $\} @$ umn.edu
}

\begin{abstract}
Despite almost 50 years of research on the use of microbubbles as ultrasound contrast agents (UCAs), the promise of high resolution dynamic perfusion imaging has not been fulfilled. This is due to the fact that the echogenicity enhancements from small clusters of bubbles in microvessels remain difficult to detect in the presence strong tissue echogenicity. A well-known pulse inversion (PI) method has been successful in exploiting the nonlinear behavior of UCAs and has led to enhanced myocardium and vascular imaging procedures. However, PI imaging has limited dynamic range due to noise amplification (fundamentally a subtraction method) We have recently proposed the use of the post-beamforming Volterra filter for separation of linear and non-linear echoes and maintaining very high dynamic range in addition to effective suppression of additive Gaussian noise. This method was shown to match or exceed the performance of PI imaging in static imaging of UCAs in flow phantoms. In this paper, we present in vivo results of UCA imaging using the cubic component of a third-order Volterra filter, together with a pixel-wise estimate of a temporal-perfusion index (TPI). We show that the TPI can be designed as a spatio-temporal estimator of the perfusion activity to provide separation between UCA activity and changes due to tissue motion due to breathing and/or pulsation. This is achieved using UCA injections with clinicallyrelevant concentrations and imaging at normal scanner setting, i.e. no sacrifice of bandwidth as may be necessary when using PI imaging. The results also demonstrate the high resolution nature of TPI imaging in both axial and lateral dimensions.
\end{abstract}

Index Terms - Nonlinear Filtering, Ultrasound Imaging and Ultrasound Contrast Agent

\section{INTRODUCTION}

Contrast enhanced ultrasound (CEUS) plays an important role in medical imaging in a variety of clinical applications. Clin-

${ }^{*}$ Funded in part by grant EB004456 from the national Institutes of Health. ical CEUS images from patients with liver tumors, for example, beautifully show hyper- or hypo-enhancements reflecting the stage or phase of the tumor when followed over time [1] in B-mode CEUS. The ultimate promise of microbubble ultrasound contrast agents (UCA) is enabling the high resolution imaging of single bubble or small clusters of bubbles in the microvasculature thus allowing for real-time perfusion imaging [2]. Despite a long history of the use of UCAs in CEUS applications, the use of UCA imaging in high resolution dynamic imaging of perfusion has not yet been demonstrated using a clinical scanner. While the nonlinear behavior of UCA microbubbles is much better understood [3], its use in imaging perfusion is still limited and its advantages are yet to be demonstrated. For example, one application area of interest that still represents a challenge is imaging blood flow in the vasa vasorum, the microvascular network providing blood supply for atherosclerotic plaques [4]. For example, molecular UCA imaging results shown in [4] using a high frequency ultrasound show that it is possible to image the vasa vasorum in a small-animal model, but this is still an elusive goal using vascular probes on clinical scanners [5].

In this paper, we present in vivo results from UCA imaging of a tumor model obtained using a clinical vascular imaging probe. The beamformed data from the linear array probe was first processed using a third-order Volterra filter (ToVF) to separate the echo signal into its linear, quadratic, and cubic components. The quadratic and cubic components were shown to produce higher specificity to UCA activity than the raw beamformed data. Log compressed image frames are computed from quadratic and cubic components to produce QB-mode and CB-mode image frames [6], respectively. A temporal measurement of intensity-adjusted intensity variance at each image pixel is then computed over a finite number of frames. This temporal perfusion index (TPI) is modified from a previously introduced measurement [6] to allow for improved spatiotemporal characterization of the perfusion at each pixel location. The results shown below demonstrate that the TPI measurement achieves significant discrimination between variations due to motion/breathing/pulsation and 
variations due to UCA activity. Furthermore, the results also demonstrate the high resolution nature of the TPI imaging.

\section{METHODS}

\subsection{In Vivo Imaging and Data Collection}

CEUS imaging was performed on a LnCAP tumor implanted and allowed to grow in the hind limb of Copenhagen rats. Several data sets were acquired at different time points and in different animals, but results from one session are presented in this paper to illustrate the ability of the TPI measurement to separate UCA activity from natural breathing and pulsation motion. The rats were anesthetized with Xylazine and Ketamine and placed in a supine position. The imaging data was collected with a linear diagnostic array probe (HST 158, Ultrasonix, Richmond, BC Canada) connected to a Sonix RP commercial ultrasound scanner with a research interface (Ultrasonix). Images were obtained before and after injection of $.75 \mathrm{~mL}$ of MicroMarker UCA (Visual Sonics, Toronto, ON Canada). Beamformed rf data was collected at $40 \mathrm{MHz}$ sampling frequency and stored for off-line processing. The data was acquired at a frame rate of 26 frames per second at a moderately low power setting in order not to destroy the UCA microbubbles. On average, 5 - 6 seconds of frame data was acquired to examine the dynamics of TPI changes over multiple heart and breathing cycles. Examples of image frames collected before and during UCA circulation are shown in Fig. 1 . The tumor is easily identifiable as a hypoechoic with oval shape.

\subsection{Nonlinear Signal Separation Using the ToVF}

We have recently described the ToVF formulation and its adaptive implementation in the context of ultrasound imaging [7]. The Volterra filter belong to a class of polynomial filters with memory [3] and offers numerous advantages in separating the nonlinear signal components. Briefly, a recursive least-squares version of the filter was implemented to:

1. Continuously update the filter coefficients for the linear, quadratic and cubic components.

2. Apply the RLS ToVF to separate the three components to form B-mode, QB-mode (quadratic), and/or CBmode (cubic) log-compressed images. For each image, the appropriate dynamic range was used in forming the intensity data assuming an 8-bit display.

For the data shown below, the dynamic range was $45 \mathrm{~dB}, 85$ $\mathrm{dB}$, and $125 \mathrm{~dB}$ for the B-mode, QB-mode, and CB-mode images, respectively. Refer to [7] for more detail about the spectral characteristics and statistical distributions of the three output components of the ToVF from both tissue and tumor regions in the presence and absence of the UCA.
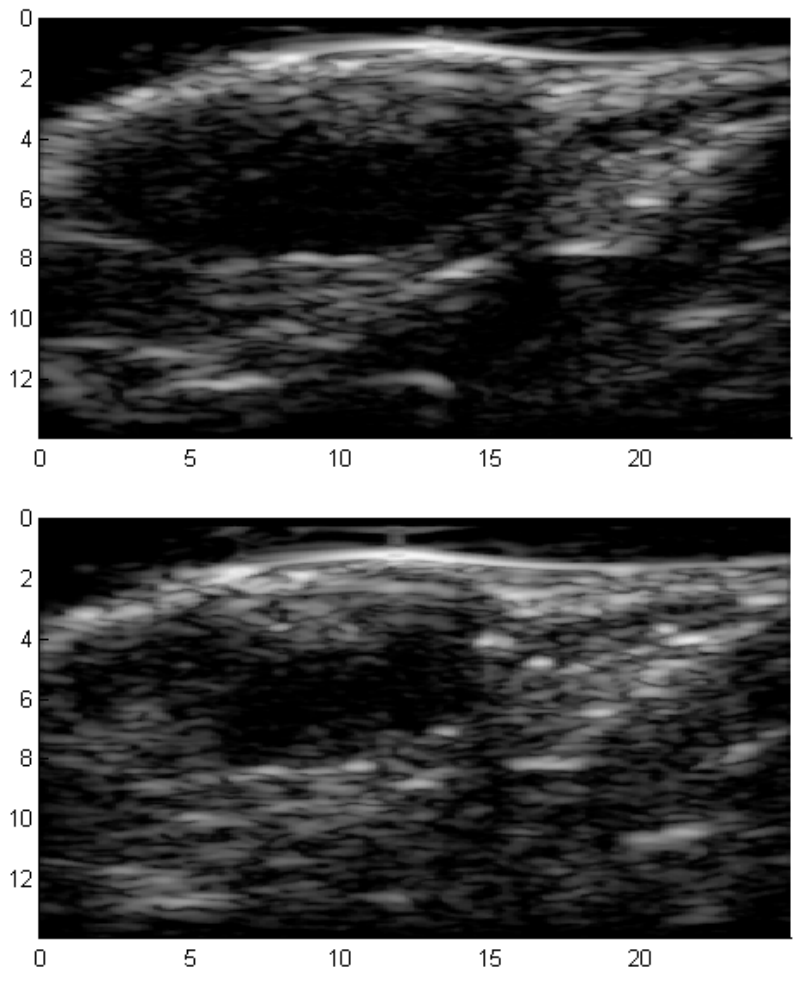

Fig. 1. Grayscale images $(50 \mathrm{~dB})$ of the tumor and the surrounding tissue without (top) and with UCA. The effect of the UCA on the echogenicity in B-mode is subtle but evident, especially in hyperechoic regions. Dimensions in $\mathrm{mm}$.

\subsection{Temporal Perfusion Index}

We have used the concept of TPI in our previous publications $[6,7]$ to capture the dynamic behavior of single bubbles or small clusters of bubbles within microvessels as they enter and leave the imaging slice, depending of the alignment between the vessel and the imaging beam. In general, the TPI can be defined as follows:

$$
T P I(x, z, k)=\frac{\sigma_{I}^{2}(x, z, k)}{\left[\hat{\mu}_{I}(x, z, k)\right]^{\gamma}}
$$

where $\sigma_{I}^{2}(x, z, k)$ is the variance of the pixel intensity at $(x, z)$ for frames $\ell=k, k+1, \ldots, k+N_{f}$. That is, the variance is computed as a point measurement over a finite number of frames. The value of $N_{f}$ can be determined from the frame rate and the range of motion observed in the B-mode image. In addition, the local mean intensity, $\hat{\mu}_{I}(x, z, k)$ is obtained through a spatial convolution in frame $k$

$$
\hat{\mu}_{I}(x, z, k)=h(x, z) * * I(x, z, k)
$$

where $h(x, z)$ is finite-support spatial averaging filter with the extent determined by the range of motion over the time $\left(N_{f}\right)$ used in computing TPI $(x, z, k)$. The parameter $\gamma$ allows for the proper normalization of the temporal variance estimate by 
the local mean. In general, for all values of $\gamma$, higher local intensities result in reduced TPI values for the same level of variance. Fractional values of $\gamma$ result in favoring the low intensities while the opposite is true for rational values of $\gamma>1$. For the case at hand, given that the echogenicity of the tumor is low, fractional values of $\gamma$ result in a good balance in TPI values in both tumor and normal tissues. For display purposes, the TPI values were scaled for an 8-bit display, i.e. covering the range of 0 to 255 . It should be noted that the use of a spatially smooth value for the local average does not compromise the spatial resolution of the TPI estimate as the variance computation is still performed on a pixel-wise basis. Still, a minimum amount of spatial filtering of the TPI estimate, especially in the axial direction, may be useful for obtaining a smooth estimate without compromise of the spatial resolution.

\section{RESULTS AND DISCUSSION}

An adaptive ToVF of order 15 was used to generate the linear, quadratic and cubic components of the beamformed echo data. The recursive least squares (RLS) algorithm was used as it was shown to produce superior results, both in terms of convergence speed and minimizing of prediction error. In what follows, we show TPI imaging results based on CB-mode imaging only, but similar results could be obtained using the other components.

\subsection{Temporal Perfusion Index}

False color overlays of the TPI maps on CB-mode images from two frames obtained before contrast injection are shown in Figure 2. The blue shade is indicative of low TPI values, below 45 on a 0 to 255 range. The nonzero TPI values reflect the sensitivity of the estimator to tissue motion and/or noise variation depending on the local tissue echogenicity. This, of course, is inevitable for any dynamic measurement based on tissue echogenicity. In the presence of UCA, however, the TPI values are significantly higher throughout the tissue region and within parts of the tumor as can be seen in Figure 3, which shows TPI maps from two frames obtained after UCA injection. The result clearly shows the TPI distribution within and outside the tumor with relatively high values at different locations in different frames, illustrating the transient and sporadic nature of the UCA-induced echogenicity change.

To further illustrate the transient nature of the TPI values in the presence and absence of UCA, we show time traces of TPI values at one pixel location (approximately $8 \mathrm{~mm}$ axially and $2.5 \mathrm{~mm}$ laterally.) This result is shown in Figure 4. This pixel was chosen because it has the highest TPI values before UCA injection, i.e. false perfusion. Note the regular structure of the estimated TPI before contrast injection. In this case, the pattern follows the breathing rhythm of the animal under anesthesia (gasps approximately every 1.5 seconds.) On the other hand, the TPI values from nearly the same location after
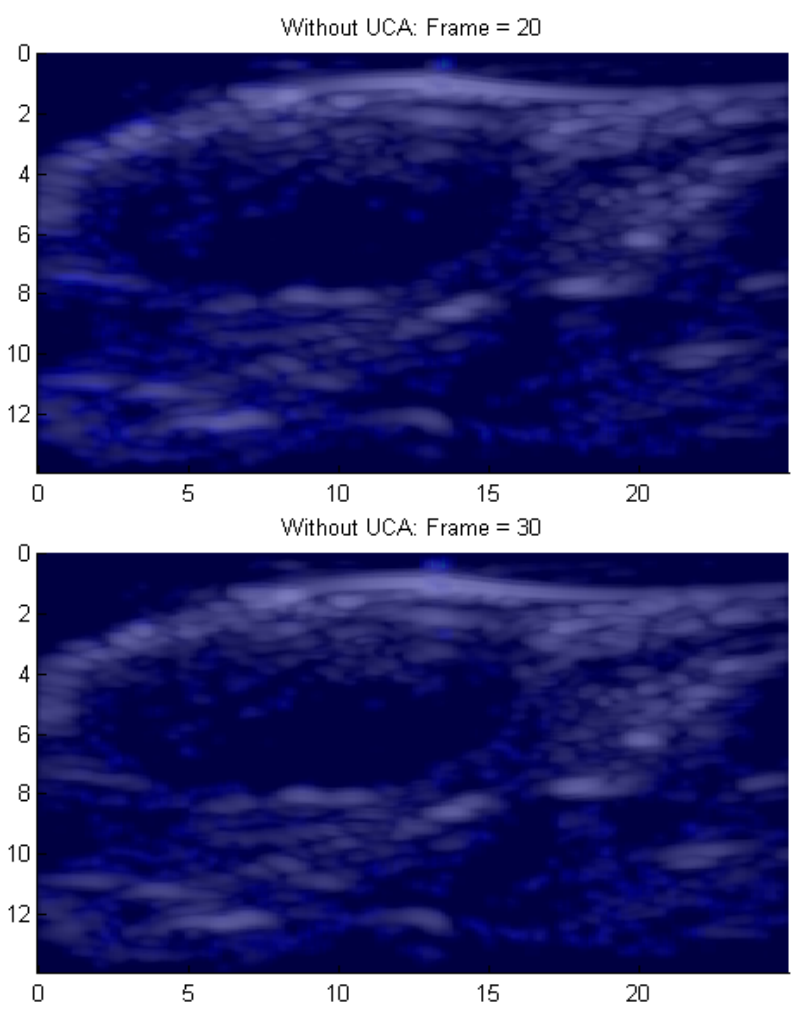

Fig. 2. TPI maps overlaid on CB-mode images for frames 20 (top) and 30 before injection of UCA. Dimensions in $\mathrm{mm}$.

contrast injection are more sporadic with significantly higher values. While this is not necessarily a quantitative measurement, it clearly shows that the pixel represents a region in the image with relatively high perfusion.

Figure 5 shows an overlay the maximum TPI value at each pixel location from all frames before (top) and after UCA injection. The result sheds some light on the underlying vascular network as one can see "trails" of enhanced TPI values within and surrounding the tumor. The relatively smaller values of TPI obtained before UCA injection can be correlated with boundaries between speckle spots or specular reflectors like the one where the traces shown in Figure 4 was obtained. The imaging results shown in Figure 5 are significant in that they reveal the heterogeneous nature of the perfusion within the tumor with the lower left quadrant well perfused compared to most of the tumor tissues.

\section{CONCLUSION}

We have presented in vivo UCA imaging results demonstrating the feasibility of dynamic estimation of UCA activity in tumor and normal tissue with a wide dynamic range of echogenicity. The TPI images clearly discriminate between echo changes due to UCA activity and changes due to tissue motion. Furthermore, the maximum TPI value projection over approximately 5.3 seconds of imaging shows clearly 

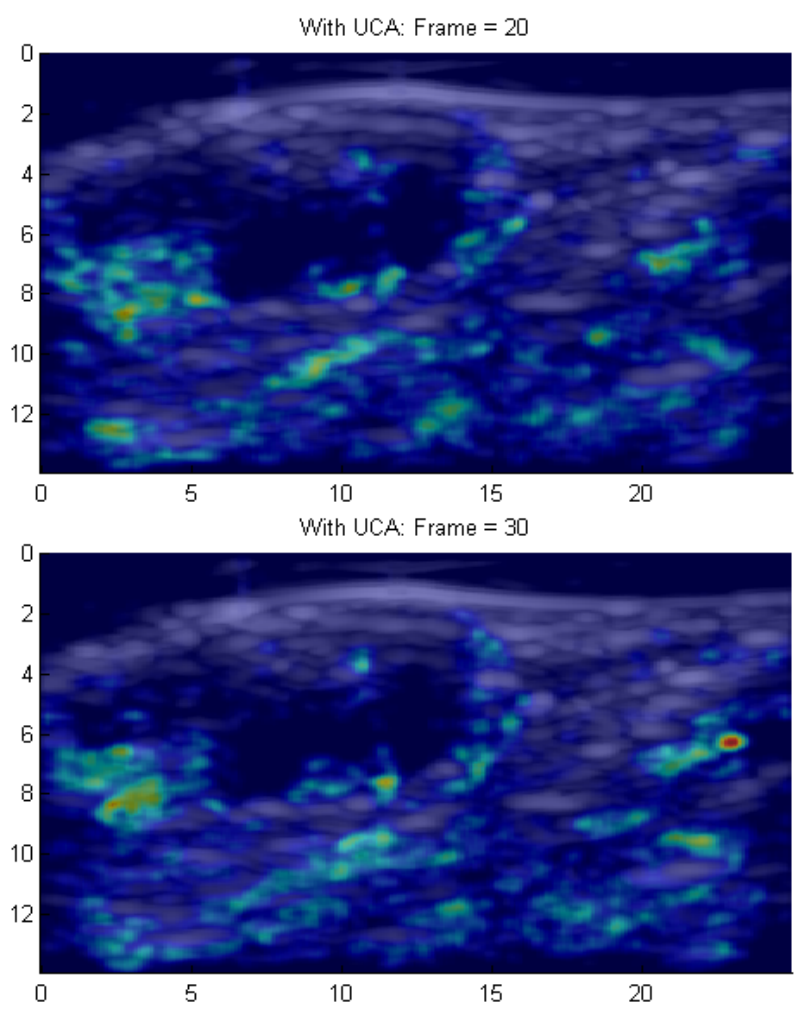

Fig. 3. TPI maps overlaid on CB-mode images for frames 20 (top) and 30 after injection of UCA. Dimensions in $\mathrm{mm}$.

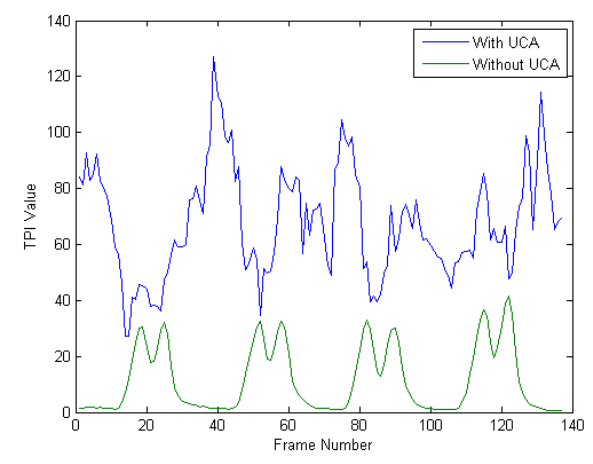

Fig. 4. TPI values at a boundary point of the tumor (approximately 8-mm axially and 2.5-mm laterally) with (blue) and without UCA.

how the heterogeneity of the perfusion pattern within the tumor. These results open the door for high resolution imaging of perfusion over a high dynamic range using standard diagnostic probes and clinically-relevant concentrations of UCA.

\section{REFERENCES}

[1] Michiel Postema and Odd Helge Gilja, "Contrast-enhanced and targeted ultrasound.," World J Gastroenterol, vol. 17, no. 1, pp.
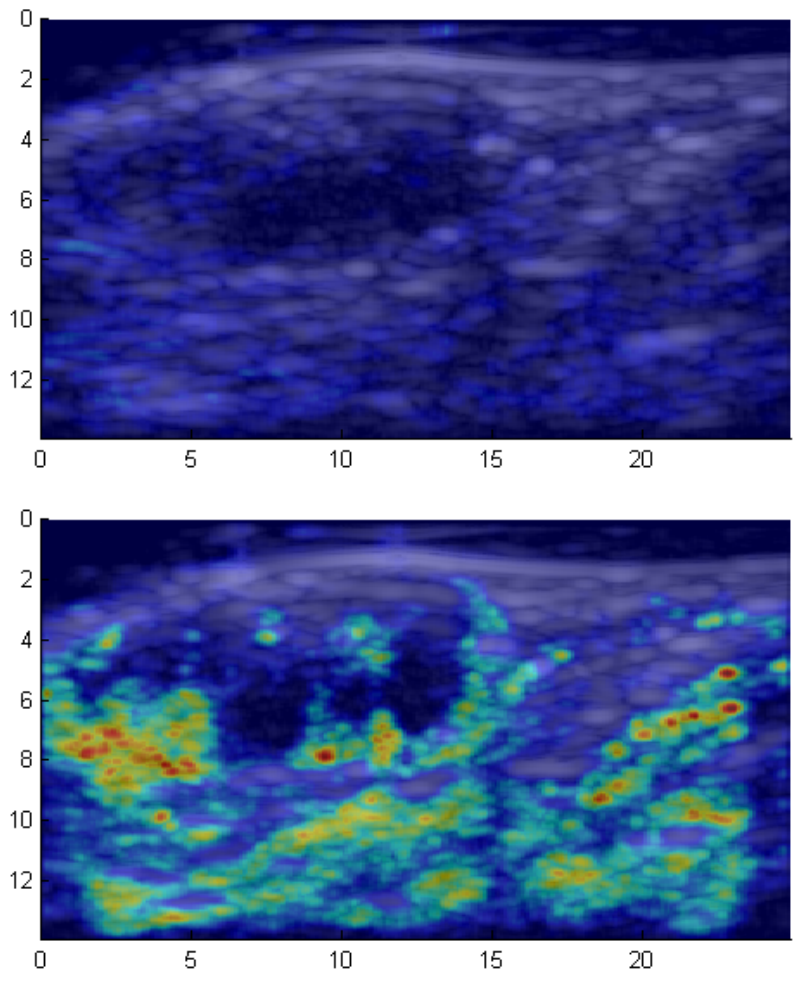

Fig. 5. Maximum TPI value maps overlaid on CB-mode images before (top) and after UCA injection. Dimensions in $\mathrm{mm}$.

28-41, Jan 2011.

[2] Nico de Jong, "Improvements in ultrasound contrast agents," IEEE Engineering in Medicine and Biology Magazine, pp. 7282, August 2002.

[3] V.J. Mathews, "Adaptive polynomial filters," Signal Processing Magazine, IEEE, vol. 8, pp. 10-26, Jul. 1991.

[4] Julius L. Decano, Anne Marie Moran, Nelson Ruiz-Opazo, and Victoria L M. Herrera, "Molecular imaging of vasa vasorum neovascularization via despr-targeted contrast-enhanced ultrasound micro-imaging in transgenic atherosclerosis rat model," Mol Imaging Biol, vol. 13, no. 6, pp. 1096-1106, Dec 2011.

[5] Theodore G. Papaioannou, Manolis Vavuranakis, Aristides Androulakis, George Lazaros, Ioannis Kakadiaris, Ioannis Vlaseros, Morteza Naghavi, Ioannis Kallikazaros, and Christodoulos Stefanadis, "In-vivo imaging of carotid plaque neoangiogenesis with contrast-enhanced harmonic ultrasound.," Int J Cardiol, vol. 134, no. 3, pp. e110-e112, May 2009.

[6] Yayun Wan, Rachana Visaria, John C. Bischof, and Emad S. Ebbini, "Quadratic B-mode and Pulse Inversion Imaging of Perfusion Defects In Vivo," Life Science Systems and Applications Workshop, IEEE/NIH, 2007, pp. 237-240.

[7] Juan Du, John R. Ballard, Jeunghwan Choi, John C. Bischof, and Emad S. Ebbini, "Adaptive Third-order Volterra Filter for Detection and Tracking of Nonlinear Oscillation in Ultrasound Echo Data," Acoustics, Speech and Signal Processing, IEEE International Conference, May 2013, pp. 1051-1055. 